\title{
TFIIH Subunit Gene
}

National Cancer Institute

\section{Source}

National Cancer Institute. TFIIH Subunit Gene. NCI Thesaurus. Code C20654.

For transcription initiation, the nonphosphorylated RNA polymerase II is recruited by TFIIF to the DB complex. This complex is recognized by TFIIE, TFIIH, and TFIIJ to form a transcription-competent complex. Phosphorylation of the C-terminal domain of the largest subunit of RNA polymerase II controls the transition from transcription initiation to elong ation and TFIIH contains a kinase activity for phosphorylating this domain. Factors that promote the association of RNA polymerase II with the preinitiation complex stimulate this activity. TFIIE, which is required for the stable association of TFIIH with the preinitiation complex, affects the processivity of TFIIH kinase. TFIIH is a multisubunit factor of at least 5 proteins of 92, 62, 43, 40, and $35 \mathrm{kD}$. A 52-kD subunit has also been identified as a component of the TFIIH core, along with p89, p62, p44, and p34. (from OMIM, LocusLink and $\mathrm{NCl}$ ) 